\title{
Development of High Sensitivity and Cost Effective Technique for the Surface Plasmon Resonance Instrument
}

\author{
M Rajavelan ${ }^{1}$, S Ananthi' ${ }^{2 *}$, and K Padmanabhan ${ }^{3}$ \\ ${ }^{1}$ Department of Network Systems \& Information Technology, Guindy Campus, University of Madras, Chennai, India \\ ${ }^{2}$ Associate Professor \& Head-in-Charge, Department of Network Systems \& Information Technology, Guindy Campus, University of Madras, India \\ ${ }^{3}$ Emeritus Professor (Hony), Alagappa College of Technology, Chennai, India.
}

Received: November 01, 2016; Accepted: November 20, 2016; Published: December 10, 2016

*Corresponding author: S Ananthi, Associate Professor \& Head-in-Charge, Department of Network Systems \& Information Technology, Guindy Campus, University of Madras, Chennai -600 025, India, Tel: +044 22202767; E-mail: ananthipradeep84@gmail.com

\begin{abstract}
Surface Plasmon Resonance (SPR) is prevalent in the field of biotechnology presently because of its label free detection of bio molecular reactions. The SPR instrument finds its place in pharmacology and drug research. SPR sensitivity is also remarkable by enabling the detection of molecules in reactions and the affinity among the groups. The theory behind the technique is well understood and attempt has been made to improve the existing technique. The instrument involves the motorized drive for the glass prism to vary the angle and the source of laser using a solar panel held against the opposite prism face, thereby catching the beam wherever it moves along that surface. The use of gold layer in the SPR instrument is the vital component that causes the SPR dip. Due to frequent use of test samples over the gold layer, the gold vanishes from the glass plate. The gradual degradation of gold layer requires frequent change of the gold slide. To solve this problem, a thin layer of lacquer coating is applied over the gold layer. But then, multiple peaks are generated than the single SPR peak. Rather than using the angle change due to Refractive Index (R.I) variations in the analyses sample, a new method of using these multiple peaks aims to integrate the multiple reflections and find their sum. This peak sum value changes in accordance with the R.I of the substance. An embedded controller (microcontroller) is used to take the signal samples and integrate them during the angle scan and to display the peak sum in each and every scan. The peak sum value is shown on an LCD interfaced to the microcontroller. An USB interface to the computer is also developed. The program using Visual Basic displays the SPR curve peaks and the related parameters on the computer screen. This enables further analysis with the laptop computer. The signals from such multiple reflections can be enhanced by an increased power laser source. This increases the sensitivity of the new technique. Thus, in this improvement to the conventional SPR instrument, we get two advantages: One is that the expensive replacement costs of the gold slide are absent. Second is the fact that more sensitive results can be obtained by amplification of the optical signals. In the conventional method based on sensitive angle variations, we cannot get any amplification effect.
\end{abstract}

Keywords: Surface Plasmon Resonance; Gold Coated Glass PlateSenor Chip; Laser Beam; Solar Cell and Multiple Reflections

\section{Introduction}

Certain important aspects on the design and operation of the SPR instrument are discussed in this paper. The SPR is a sensitive method for optical Refractive index change based analytical techniques and finds importance in bimolecular work, drug synthesis, protein to protein affinity and fine measurements of Nano-material properties $[1,2]$. The instrument is a complex combination of optical, electromechanical, and electronic and microprocessor based devices [3, 4]. It requires only very minute quantities of the substances analyzed. Currently available instruments are very sophisticated and expensive.

It is felt necessary to design and develop a moderately simple enough instrument which can serve the above purposes with some additional features incorporated in it. In every aspect of the fabrication of the instrument, special attention was given to choose parts, materials and circuitry to minimize cost and boost sensitivity. The several improvements that have been experimented and studied include the optical parts [5], the electromechanical components and assembly, the detector and amplification of the signals, the processing and display of the signals as well as the choice of a suitable microcontroller to handle the operation of the entire instrument. The development of suitable software for a laptop to be associated with the set up was also an additional involvement.

To illustrate the various aspects of its design, the figure 1 gives an essence of the technique [6]. Its main component is a right angled BK7 glass prism. The laser source of red radiation with a suitably polarized light to fall on the broad face of the prism at varying angles of incidence is the next important optical component and assembly. The detection of the reflected light signal and transuding the same to a voltage is the next one. The angulations signal processing and evaluation are the aspects to be dealt with by the embedded controller $[7,8]$. 


\section{Methods}

\section{Geometrical alignment for optimal ray positioning}

In many of the designs, the angle of incidence of laser beam is varied over a range of angles. In studying kinetics of fast bio reactions through Refractive Index (R.I) changes, if we cannot find the R.I. at a specific position on the liquid film, it will amount to finding only an average change in R.I. over the length of the film. Usually, the film is made to move slowly by the capillary injection pump.

Therefore a small distance movement will amount to a time over which considerable reaction change might have taken place. Therefore, the reaction kinetics curve for a fast reaction with peak will flatten out in the measurement if the point does not coincide at the same position of the hypotenuse at all angles. So the accuracy in measuring the molecular sample will deviate by a large amount. In the (figure 2) it is shown that when the turning beams carrying the laser rotates with the centre of rotation at the table centre (where the prism is kept), for varying angles, the point of incidence of the laser moves along the hypotenuse of the prism-1, to 2 to 3 . That will make the reading taken of the reflected light correspond to different positions on the hypotenuse and therefore from different points on the solution film that passes over the same. That may not find the R.I. at a specific point on the film of solution. That means we have to ensure that the point of laser light falling on the prism remains steady as the prism is turned in angle to vary the angle of incidence. Generally, the instruments designed do not seem to be aware of this critical problem of spot movement over the slide as the angle of incidence is varied. This is illustrated in figure 2 where at three angular values, the spot falls on different spots on the gold slide. Thus it has been found that some importance should be bestowed on the optical geometry design for SPR used for fast reaction detection.

\section{Solution to optical geometry problem of SPR setup}

Suppose $\mathrm{C}$ is the Centre of rotation of the rod which rotates over an angle range carrying the laser beam. Then, if all rays should converge exactly at the centre of the prism hypotenuse, then, what is the relative position of the prism w.r.t. the point C? The solution to this problem is an optical Geometry problem. This problem was solved using a computer program. That program found out the optimal position of the rotating centre of the turn table. So in order to increase the accuracy in the measurement of molecular samples, it should be made that laser beam converges at the centre of hypotenuse of the prism. The rotation centre is the point where the green lines meet within the prism.

We have observed that in order to make the rays meet at one and the same point on the glass gold plate, it is required to determine the rotation centre to hypotenuse centre offset distance in the $\mathrm{x}$ and $\mathrm{y}$ directions. For this purpose, a computer program has been developed and the result of the program is just concisely shown in the figure 3 , indicating the method of estimating these offset distances. The prism on the turn table is thus positioned accordingly.

\section{Graphical method for positioning the optimal centre of rotation}

By using a Graphics program, starting from point P, rays are drawn using graphics line draw Commands. Rays as shown in Red color are these internal rays. Each red ray coming down meets the left small side of the prism. At this point, applying Snell's law, the ray on the air side is drawn. For each ray, there is a ray coming to the prism from the light source (laser) (figure 4). Now, extending each ray further to the right, there is a meeting point of all such rays. That point is marked as C. This is the Centre of rotation for the prism to turn. Keeping the laser beam fixed, if

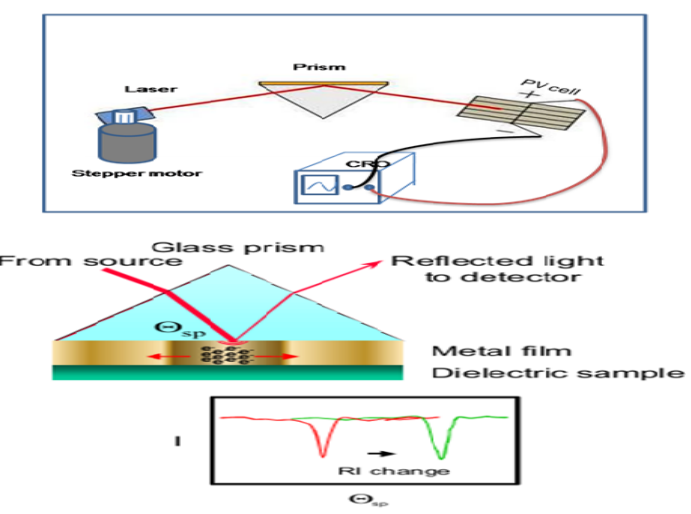

Figure 1: The schematic of the Surfacce Plasmon resonance based measurement system

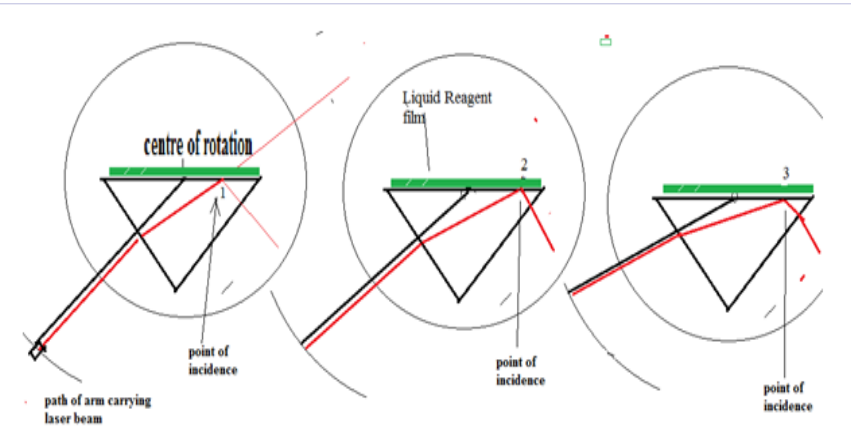

Figure 2: When the turning beam varying the laser rotates with the centre of rotation at the table centre (where the prism is kept), for varying angles, the point of incidence of the laser moves along the hypotenuse of the prism- 1 , to 2 to 3 .
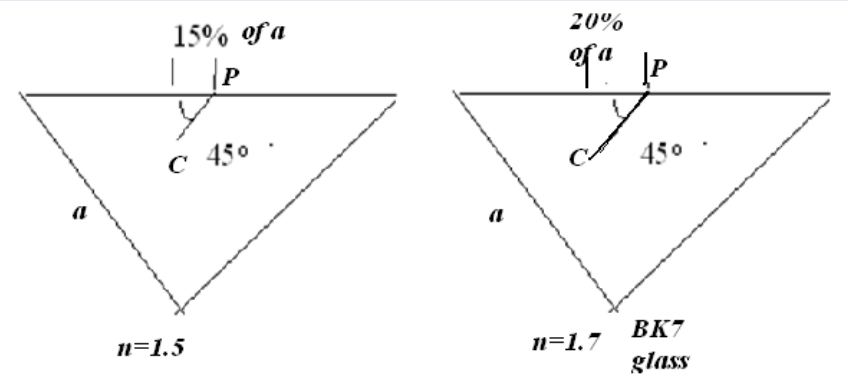

Figure 3: The Centre of Rotation is marked inside the Prism for two different prisms. 
the prism is turned over these angles, then the rays will all meet at the point P.? Thus, a method has now been found to meet the requirement that the light spot on the hypotenuse face stays at one and the same point for all angles of incidence.

At all angles, the laser beam hits at the centre of hypotenuse of the prism.

\section{Experimental set-up}

In the design of the rotation of the motor carrying the laser, the stepper motor has to be driven by a suitable gear box of a good ratio. A 30:1 ratio with a 200 steps per rotation stepper motor gives an angular change of $360 /(200 \times 30)=0.06$ degree per step. By suitably using gear ratios, this can be enhanced to $1 / 100^{\circ}$ at the most.

The drive for the stepper motor is arranged by a four wire interface from an embedded controller and a suitable four transistor driver circuit shown in the (figure 5).

Thus four different types of SPR experimental setups have been developed for a satisfactory result. These are as under:

SPR design setup I: Fixed prism and rotating laser source,

SPR design setup II: Rotating prism and fixed laser source,

SPR design setup III: Rotating prism and detector with fixed laser source and goniometry -2 for detector

SPR design setup IV: Modified experimental setup II (Rotating prism with geared stepper motor and fixed laser)

The SPR design setup IV is well designed as the final SPR instrument in which certain reasonable features are embedded. It has sensitivity and good angular resolution. (Figure 6) illustrates the same.

The SPR reflected signal dip is constant and only angle changes with Specimen R.I. (figure 7). The dip in the curve of reflected light intensity happens at an angle $\theta$ Plasmon and this angle is characteristic of the sample kept adjacent to the gold film or metal. Actually, this angle is dependent on the R.I. values of prism, the gold and the sample. But when the R.I. of the sample changes, the dip magnitude does not change but only the angle at which the dip occurs changes.

The gold coated specimen glass plate and other sanitized biochips:

There are two types of gold coated glass plates.

1. Pure gold coated over the glass plate using Sputter coater

2. Gold coated over the glass plate with TiO2.

Gold layer is very important to generate the surface Plasmon, so it is necessary to coat the gold layer over the surface of the glass plate and place it over the hypotenuse of the prism without any air gap. The simplest and economical way of coating gold layer over the glass plate is by using a Sputter Coater with a gold anode $50 \mathrm{~nm}$ of pure gold layer is coated for our purpose and several such slides are prepared and stored in a vacuum chamber. Sputter coaters are available with thickness monitoring facility.



Figure 4: Showing the convergence of rays internal (C) in the prism while the actual point $\mathrm{P}$ is one and the same for all angles. Prism table rotation centre will therefore be Point C

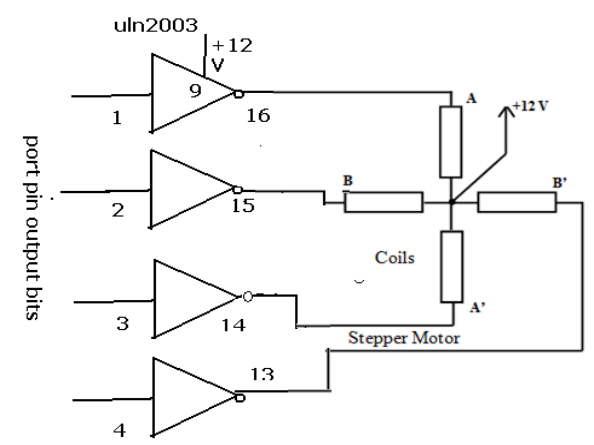

Figure 5: ULN 2003A driver IC for stepper motor.

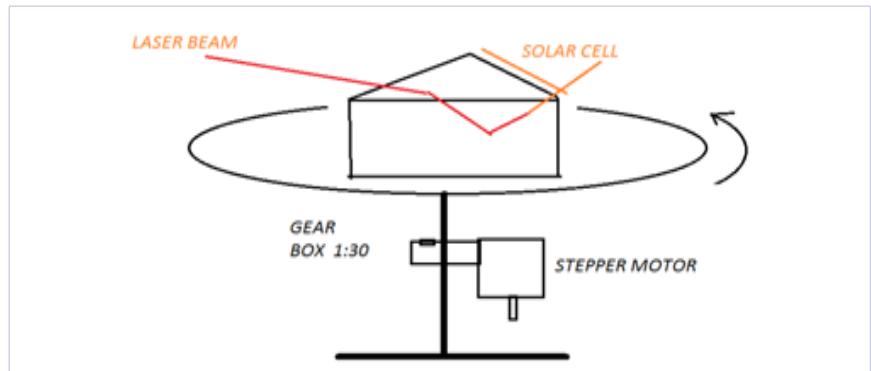

Figure 6: The prism on the turn table driven by gear from stepper motor.

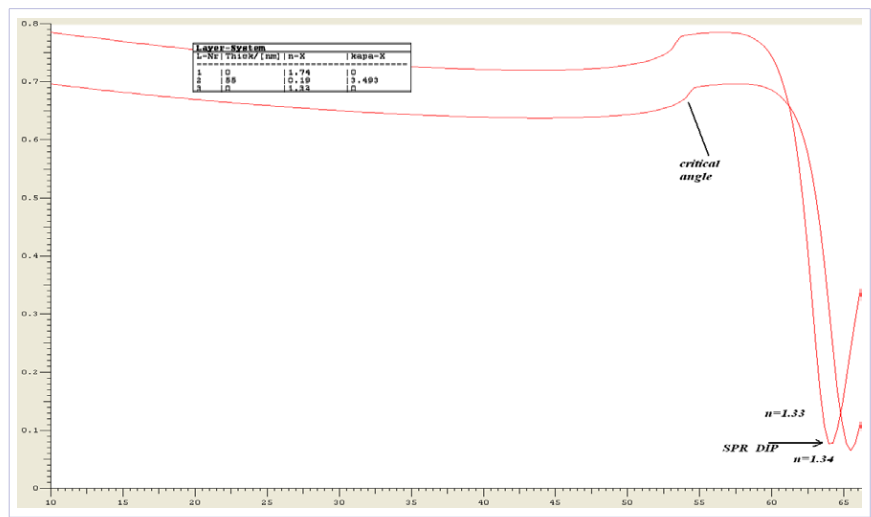

Figure 7: For substance R.I. values 1.33 and 1.34, the angle change is from 66 to 64 degrees. The amplitude of the dip is not varying much. 
The pure gold coated over the glass plate may be washed off quickly within a few sample tests. This may be overcome to some extent by using an adhesive layer like copper or titanium that is coated over the glass plate before the gold is plated. The gold with Titanium Oxide coated over the glass plate is available from vendors dealing with SPR instruments.

When it is required to study bimolecular compound interactions, it is necessary to apply a suitable ligand coating on the gold surface. BiaCore Co. sells sensitized gold plates with Dextran, CM5 and some other chemicals. Preparation of gold plated slides with such sensitized coatings can be prepared by the analyst as and when needed.

The main running cost of the instrument lies in the expensive gold coated slide. In using the solar cell, the two cells are connected in series electrically. Each solar cell has a positive (+) and a negative $(-)$ terminal. The two are joined and the two flat cells are kept adjacent to the second reflecting face of the prism. For He-Ne Laser, when the light incidence is such that the ray is totally reflected internally, the light beam directly impinges on the solar cell. The point of impingement may change as the incidence angle changes due to prism turning on the round table. But the ray is not missed for any angle. The D.C. signal voltage that is got is about $0.5 \mathrm{~V}$ then. When there is surface Plasmon resonance at the particular angle, the light diminishes in intensity. Then, the voltage drops to anywhere between 0.3 to $0.4 \mathrm{~V}$. Thus, with the Helium Neon laser of $10 \mathrm{~mW}$, we can get about 100-200 $\mathrm{mV}$ change in signal. This signal is to be amplified. For the $50 \mathrm{~mW}$ diode laser, we get $5 \mathrm{~V}$ for full ray impingement and about 3-4 V for SPR condition. Thus, the voltage change we get is about 1 to 2 $\mathrm{V}$, quite a sizeable value.

\section{Flow cell for analytes design}

In general, the flow cell is a small compartment like arrangement used to contain the samples to be tested. It is placed over the prism surface after the gold coated glass plate in contact with prism. The gold coating will be towards the flow cell. The leading SPR instrument developers have used different types of flow cells for different applications. But the most frequently used type of flow cell is PDMS (Poly-DiMethyl-Siloxane) flow cell of volumes of $1 \mu \mathrm{l}, 5 \mu \mathrm{l}, 100 \mu \mathrm{l}$ and they are used as standard flow cells in general. Also the SPR instrument developers made use of custom flow. A suitable via media design of a flow cell which was used for the set up constructed.

\section{The design with the embedded controller}

A PIC 18F2550 microcontroller based embedded board has been developed and it is programmed in such a way that it supplies the pulses for the stepper motor movement and acquires the data on its ADC channel. The data is transferred to the computer and plotted as the SPR signal in a picture window. The computer has Visual basic software developed for the purpose of operating the instrument. All software, both for the firmware of the PIC 2550 as well as the Visual Basic based computer program was developed. Hence the operation is made user friendly from the computer. The PIC2550 communicates with the computer through the USB port.
By feeding the number of steps to be turned on the computer, and by clicking start icon in the screen, the stepper motor starts, turns and the fixed laser source shines over the surface of the prism, gold layer and through the sample. After completion of the number of steps of rotation the stepper motor stops. Now, by clicking the read icon on the laptop, the reflected signal measured by the ADC channel is stored in the PIC microcontroller and transmits to the computer via the USB interface. Then, the data is plotted for the values corresponding to the SPR dip. The data is stored as a file in a folder. By loading this file in Mat lab, it has been possible to make further data analysis. The figure shows the real time plot of SPR in the laptop from the SPR instrument prototype.

\section{Results}

The picture of the typical fabricated instrument with all the features is shown in figure 9. The table below illustrates the details of the optical and other components shown in the table 1. With respect to the refractive index of material the SPR dip changes. The figure $8 \mathrm{a}$ and $\mathrm{b}$ shows the generated surface Plasmon resonance is measured at CRO and plotted in laptop computer screen through microcontroller and visual basic GUI. The figure 8c shows the SPR dip for two different dielectric samples and change in dip occurs. The change in SPR dip is the amount of binding of ligand and analyte sample measured.

\section{Conclusion}

A portable, low cost and high sensitivity SPR instrument is constructed and developed for bio molecular interaction studies and assessment applications. This will include the building up a typical instrument, its hardware, associated software programs as well as the computer interface. The associated techniques and algorithms for interpretation of results. Optical ray positioning for steady and accurate measurement of bio molecules is made for increasing the sensitivity in the measurement of samples. The higher cost Photo diode array is replaced by low cost, high sensitive solar cell. This considerably reduced the making cost and running cost of the instrument. Thus, sensitivity and resolution are quite good when compared to commercial SPR instrument. Thus, this SPR technique has been experimentally verified in our investigations.

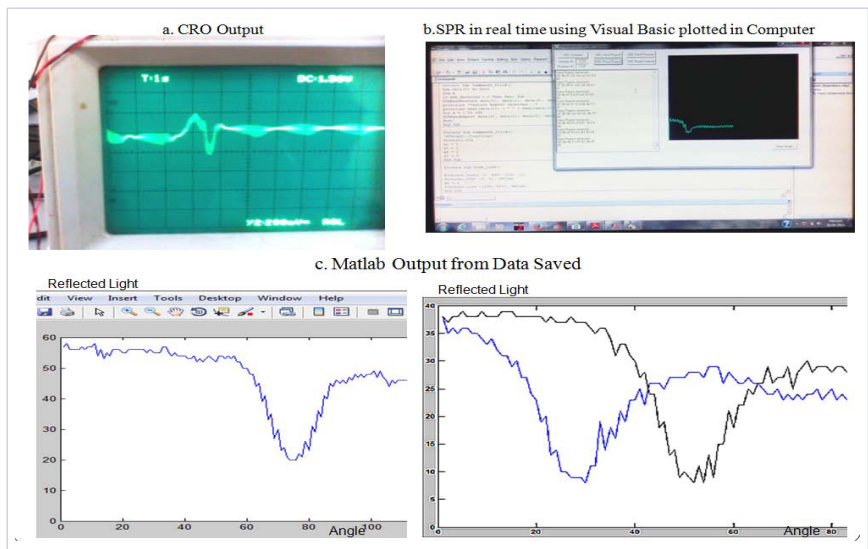

Figure 8: Shows a). SPR Dip in CRO, b). SPR Dip in the laptop screen, c). SPR curve for two different samples 


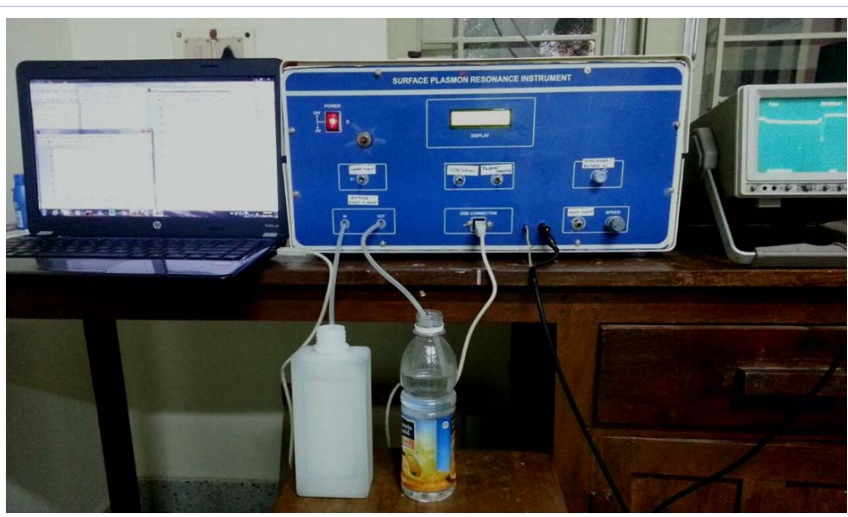

Figure 9: Shows Photograph of developed Surface Plasmon Resonance instrument.

Table 1: Components used for the surface plasmon resonance.

\begin{tabular}{|l|l|l|l|}
\hline Materials & Thickness, nm & $\begin{array}{l}\text { Refractive } \\
\text { index, n }\end{array}$ & $\begin{array}{l}\text { Absorption } \\
\text { Coefficient, } \mathbf{k}\end{array}$ \\
\hline BK7 Prism & 0 & 1.714 & 0 \\
\hline Matching oil & 1000000 & 1.714 & 0 \\
\hline Glass plate & 5000000 & 1.714 & 0 \\
\hline Gold layer & 50 & 0.1995 & 3.6425 \\
\hline $\begin{array}{l}\text { Water(as } \\
\text { sample) }\end{array}$ & 0 & $1.33 \& 1.35$ & 0 \\
\hline
\end{tabular}

\section{References}

1. Richard BM, Schasfoort, Anna J Tudos. Hand book of SPR Technology. Royal Society of Chemistry. RSC Publishing. 2008.

2. Homola J. Surface plasmon resonance sensors for detection of chemical and biological species. Chem Rev. 2008;108(2):462-493.

3. Padmanabhan K, Ananthi S. A Treatise on Instrumentation Engineering. IK International publication. 2011.

4. Moreira CS, Barreto Neto AGS, Lima Neff HAMN. Features and application of a microcontroller-driven auto sampler applied to a surface plasmon resonance biosensor platform. VIII Semetro. João Pessoa, PB, Brazil. 2009;17-19.

5. Sreekumaran Nair A, Renjis Tom T, Rajeev Kumar R, Subramaniam Pradeep C. Chemical Interactions at Noble Metal Nano Particle Surfaces-Catalysis,Sensors and Decices. COSMOS. 2007;3(1):103-124.

6. Bao Z, Jiang GLD, Cheng W, Ma X. ZnO sensing film thickness effects on the sensitivity of surface plasmon resonance sensors with angular interrogation. Materials Science and Engineering B. 2010;171:155158.

7. Muhammad Kashif, Ahmad Ashrif Bakar A. Norhana Arsad, Sahbudin Shaari. Development of Phase Detection Schemes Based on Surface Plasmon Resonance Using Interferometry. Sensors. 2014;14(9):15914-15938. doi:10.3390/s140915914

8. Gaurav Gupta, Jun Kondoh.Tuning and sensitivity enhancement of surface plasmon resonance sensor. Sensors and Actuators B: Chemical. 2007;122(2):381-388. 